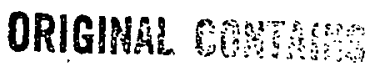

calot inger:

Ionthly Mean Large-Scale tnalyses of Upper-Tropospheric Humidity and Wind Field Divergence Derived from Three Jeostationary Satellites
Johannes Schmetz, W. Paul Menzel, Christopher Velden, Xiangqian Wu, Leo van de Berg, Steve Nieman, Christopher Hayden, Kenneth Holmlund, and Carlos Geijo 


\section{Monthly Mean Large-Scale Analyses of Upper-Tropospheric Humidity and Wind Field Divergence Derived from Three Geostationary Satellites}

\section{Abstract}

This paper describes the results from a collaborative study between the European Space Operations Center, the European Organization for the Exploitation of Meteorological Satellites, the National Oceanic and Atmospheric Administration, and the Cooperative Institute for Meteorological Satellite Studies investigating the relationship between satellite-derived monthly mean fields of wind and humidity in the upper troposphere for March 1994. Three geostationary meteorological satellites GOES-7, Meteosat-3, and Meteosat-5 are used to cover an area from roughly $160^{\circ} \mathrm{W}$ to $50^{\circ} \mathrm{E}$. The wind fields are derived from tracking features in successive images of upper-tropospheric water vapor (WV) as depicted in the $6.5-\mu$ absorption band. The upper-tropospheric relative humidity (UTH) is inferred from measured water vapor radiances with a physical retrieval scheme based on radiative forward calculations.

Quantitative information on large-scale circulation patterns in the upper troposphere is possible with the dense spatial coverage of the $W V$ wind vectors. The monthly mean wind field is used to estimate the large-scale divergence; values range between about $-5 \times 10^{-6}$ and $5 \times 10^{-6} \mathrm{sec}^{-1}$ when averaged over a scale length of about $1000-2000 \mathrm{~km}$. The spatial patterns of the UTH field and the divergence of the wind field closely resemble one another, suggesting that UTH patterns are principally determined by the large-scale circulation.

Since the upper-tropospheric humidity absorbs upwelling radiation from lower-tropospheric levels and therefore contributes significantly to the atmospheric greenhouse effect, this work implies that studies on the climate relevance of water vapor should include threedimensional modeling of the atmospheric dynamics. The fields of UTH and WV winds are useful parameters for a climate-monitoring system based on satellite data. The results from this 1-month analysis suggest the desirability of further GOES and Meteosat studies to characterize the changes in the upper-tropospheric moisture sources and sinks over the past decade.

"European Organization for the Exploitation of Meteorological Satellites, Darmstadt, Germany.

+NOAA/NESDIS, Madison, Wisconsin.

"Cooperative Institute for Meteorological Satellite Studies, University of Wisconsin-Madison, Madison, Wisconsin.

- European Space Agency, European Space Centre, Darmstadt, Germany.

Corresponding author address: Dr. Paul Menzel, NOAA/NESDIS, 1225 West Dayton St., Madison, WI 53706.

In final form 26 January 1995.

C1995 American Meteorological Society

\section{Introduction}

The water vapor band of the geostationary satellites is routinely used for extracting upper-tropospheric humidity (UTH) and water vapor (WV) wind fields. Apart from the utility of both products in numerical weather prediction and model validation, it has become evident that UTH and WV winds constitute an important source of climatological information. Only a geostationary platform provides measurements of water vapor amount and motion on a large scale from a single observing system; thus, these measurements serve as truly independent datasets for comparisons with other climatologies. Through joint efforts at the European Space Operations Center (ESOC) and the Cooperative Institute for Meteorological Satellite Studies (CIMSS), data from three geostationary satellites (GOES-7 at $112^{\circ} \mathrm{W}$, Meteosat-3 at $75^{\circ} \mathrm{W}$, and Meteosat-5 at the Greenwich Meridian) are processed for March 1994 with similar algorithms to derive UTH. and $W V$ winds covering $160^{\circ} \mathrm{W}-50^{\circ} \mathrm{E}$ (Fig. 1). Monthly mean observations are computed from two UTH and WV wind fields per day (1200 and 0000 UTC) for GOES-7 and Meteosat-3; for Meteosat-5 eight UTH and four WV wind fields are used per day.

\section{Importance of UTH}

Satellite determinations of UTH have been shown to be useful for deriving climatologies (van de Berg et al. 1991; Wu et al. 1993) that can be compared with those derived from models (Del Genio et al. 1994; Stephens et al. 1993; Soden and Bretherton 1993; Schmetz and van de Berg 1994). The comparisons reveal significant differences between UTH from satellite observations and those in general circulation models.

Figure 2 illustrates that all altitude levels of the watervapor profile, including those represented by the 


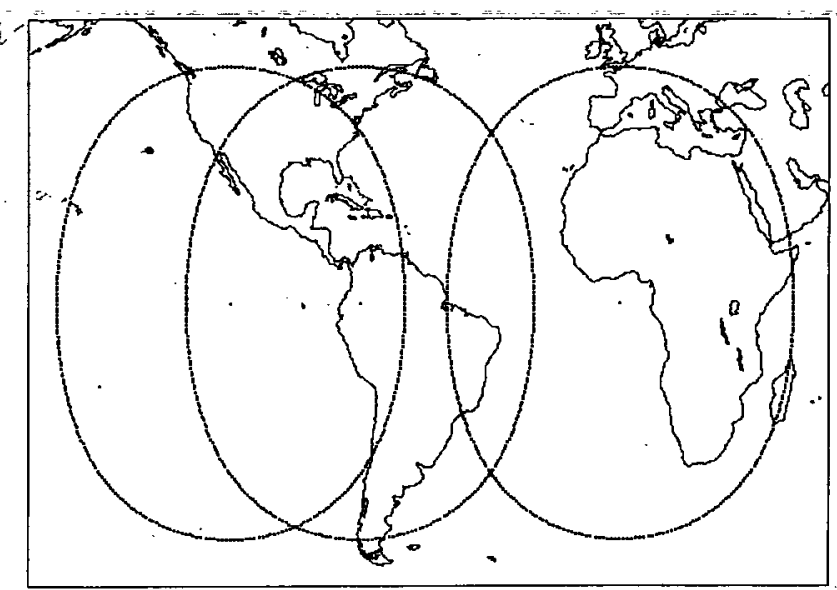

FIG. 1. Coverage from the three geostationary satellites (GOES-7 at $112^{\circ} \mathrm{W}$, Meteosat-3 at $75^{\circ} \mathrm{W}$, and Meteosat -5 at the Greenwich Meridian) out to $50^{\circ}$ from nadir.

UTH, are important to the water vapor greenhouse effect. The greenhouse effect (e.g., Inamdar and Ramanathan 1994) is defined here as the difference between the longwave flux emitted by the surface and the outgoing radiative flux (OLR) escaping from the top of the atmosphere. A radiative transfer model is used to compute the change of the OLR as a function of perturbations in the vertical water vapor profile. A $10 \%$ increase in relative humidity is added to a standard tropical profile at a single level at a time; the levels are at 1-, 3-, 5-, 7-, 9-, 11-, and 13-km altitudes. The sensitivity is expressed as the decrease in OLR divided by the increase in total precipitable water, $\mathrm{del}(\mathrm{OLR}) / \mathrm{del}(\mathrm{PW})$. Figure 2 shows that this sensitivity increase from about 2.8 to about $1200 \mathrm{~W} \mathrm{~m}^{-2}\left(\mathrm{~g} \mathrm{~cm}^{-2}\right)^{-1}$ as the $10 \%$ relative humidity perturbation is shifted from 1- to 13-km altitude (solid curve). A mixing ratio profile typical for a tropical atmosphere is also shown in Fig. 2 (dashed curve). The greenhouse effectiveness of a single $\mathrm{H}_{2} \mathrm{O}$ molecule (solid curve) rapidly increases with altitude. This is to be expected since radiation from the lower atmosphere absorbed by $\mathrm{H}_{2} \mathrm{O}$ molecules high in the troposphere is reemitted at colder temperatures, in contrast to reemission at warm temperature by the lower-atmospheric $\mathrm{H}_{2} \mathrm{O}$ molecules. The exponential decrease of the water vapor with altitude balances the increase of the greenhouse effectiveness of a single water vapor molecule. The combined effect suggests that the sensitivity of OLR to perturbations in the water vapor profile in a tropical atmosphere is roughly constant up to an altitude of -about-10-km--Thus,-Fig:-2-indicates-the-need-forobservations of the UTH, as well as observations of total precipitable water (which is dominated by lowlevel water vapor) in studies of the atmospheric greenhouse effect.

\section{UTH algorithms}

\section{a. Meteosat-5}

The UTH is inferred from the WV band $(5.7-6.9 \mu)$, which has a spatial resolution of $5 \mathrm{~km} \times 5 \mathrm{~km}$. The WV band observes the earth's atmosphere in a spectral region characterized by strong $\mathrm{H}_{2} \mathrm{O}$ absorption and is sensitive to approximately the upper $3 \mathrm{~mm}$ of total water vapor (Schmetz and van de Berg 1994). In a cloud-free atmosphere, this WV band senses typically down to a pressure level of $500-600 \mathrm{hPa}$. The height allocation of the broad contribution function depends on the actual profiles of temperature and humidity and the satellite viewing angle (Fischer et al. 1981).

The UTH retrieval follows the approach described by Schmetz and Turpeinen (1988). Radiative forward calculations are performed for different relative humidity profiles. The necessary temperature profiles are taken from short-term model forecasts provided by the European Centre for Medium-Range Weather Forecasts. The different relative humidity profiles are constructed as follows: below $600 \mathrm{hPa}$ the forecast profile is used, between 600 and $300 \mathrm{hPa}$ the relative humidity is fixed at one of six values $(1 \%, 5 \%, 10 \%$, $20 \%, 40 \%, 100 \%$ ), and above $300 \mathrm{hPa}$ the relative humidity decreases linearly to $0 \%$ at $100 \mathrm{hPa}$. Comparisons of the UTH retrieval (van de Berg et al. 1991) with radiosondes show a root-mean-square difference of less than $10 \%$. The Meteosat-5 UTH calculations use clear-sky radiances obtained from a multispectral image classification for segment areas (32 $\times 32$ pixels), ranging from 160 up to $230 \mathrm{~km}$ on a side.

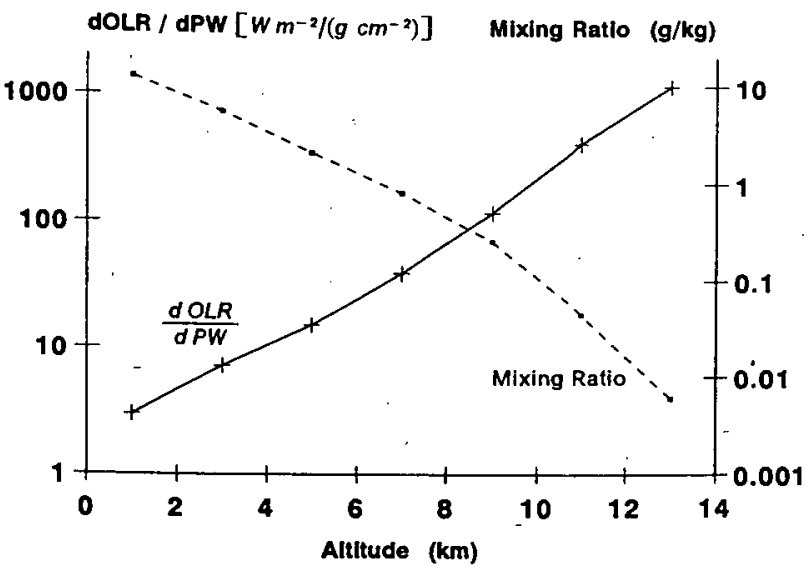

FIG.2. Sensitivity of the outgoing longwave radiative flux density (OLR in $W^{-2}$ ) to changes in precipitable water ( $P W$ in $\mathrm{g} \mathrm{cm}^{-2}$ ). PW has been varied at different levels, and the sensitivity is expressed as the decrease in OLR over the increase in PW. The dashed curve corresponds to the mixing ratio profile characteristic of a tropical standard atmosphere. 
The Meteosat-5 UTH in this study is somewhat different from that presented in van de Berg et al. (1991). That work confined valid UTH retrievals to segment areas that were totally free of medium- and high-level cloud so that the UTH was representative of the whole segment. Here the UTH retrieval is extended to segments that are partially covered with high- and medium-level cloud by using the $W V$ radiances from the portion of the segment that contains clear sky. This tends to moisten the UTH, especially in the tropical convective regions where high-level clouds prevail.

The operational calibration of the Meteosat WV band is based on radiative transfer calculations with radiosonde profiles and collocated clear-sky $W V$ observations. A recent recalibration algorithm has been added for this study, which improves calibration performance (Schmetz and van de Berg 1994).

\section{b. Meteosat-3}

Meteosat-3 data are similar to those of Meteosat-5, differing only in spectral range (5.8-7.3 $\mu$ ). However, the UTH retrieval from Meteosat-3 differs from the Meteosat-5 UTH retrieval in several aspects. The major difference is that the Meteosat-3 UTH is retrieved for each pixel regardless of cloud conditions, so it has much higher spatial resolution. When the WV radiance for a given pixel is affected by cloud, the UTH does not represent a column-mean relative humidity and can indicate up to $100 \%$ relative humidity. Including cloudy pixels in the retrieval increases the monthly. mean UTH by about $10 \%$ (absolute) in convective areas; no change is observed in areas without highlevel cloud. As a result, monthly mean Meteosat-3 UTH values are slightly more moist than those from Meteosat-5 in cloudy regions.

There are also several minor algorithm changes. Soden and Bretherton (1993) showed that, under normal conditions, the WV band brightness temperature is close to a logarithmic function of the UTH. Accordingly, the forward calculation of UTH from Meteosat-3, which is used to generate the lookuptable for WV vapor band brightness temperature, is now performed on a different set of values $(1 \%, 5 \%, 15 \%$, $30 \%, 60 \%, 100 \%$ ) and the interpolation is changed from linear to loglinear. Finally, the CIMSS forward model is used for radiative transfer computation.

\section{c. GOES-7}

The GOES-7 WV band $(6.4-7.1 \mu)$ has a spatial resolution of $7 \mathrm{~km} \times 7 \mathrm{~km}$ at nadir. While the UTH retrieval algorithm used with GOES-7 is identical to that used with Meteosat-3, the UTH fields from the two satellites are somewhat different because the two instruments sense in a slightly different part of the spectrum. In a clear atmosphere, the GOES-7 WV band typically senses down to a pressure level of 400-500 $\mathrm{hPa}$, depending on view angle and atmospheric moisture content (Hayden 1988). Thus, compared to the Meteosats, the GOES-7WV band senses radiation from a slightly higher layer in the upper troposphere. For March 1994 over an area $\left(30^{\circ} \mathrm{N}-\right.$ $30^{\circ} \mathrm{S}, 82^{\circ} \mathrm{W}-105^{\circ} \mathrm{W}$ ) with relatively equal and small viewing angles for both GOES-7 and Meteosat-3, tests showed the GOES-7 UTH was typically $5 \%$ higher than the Meteosat-3 UTH.

The empirical distribution function (EDF), described by Weinreb et al. (1989), is employed to eliminate this intersatellite bias. For each of the concurrent observations from the above-mentioned area (62 for March), an EDF is constructed that is used to adjust the GOES-7 UTH to have the same statistical distribution as the Meteosat-3 UTH. The unadjusted GOES-7 $\mathrm{UTH}$, which is representative of a higher layer in the upper troposphere, is consistently more moist than the Meteosat-3 UTH. This suggests that the relative humidity for the period and area under consideration increases with height in the upper troposphere. Although such profiles are not uncommon, it is generally agreed that relative humidity decreases with height for longer-term averages. A possible explanation is that Meteosat-3 calibration was found to be warm with respect to GOES-7, which would make the UTH drier (Menzel et al. 1994). Further investigation is required to clarify this issue.

\section{d. Mean UTH for March 1994}

Figure 3 shows the monthly mean UTH for March 1994 from all three satellites. The adjusted GOES-7 UTH merges very well with the Meteosat-3 UTH without noticeable discontinuity, while the transition from Meteosat-3 to Meteosat-5 is distinguishable (primarily because different UTH retrieval procedures are used). A unified retrieval for all satellites would likely alleviate these boundaries.

Highest relative humidities of about $60 \%-70 \%$ are observed over the tropical convective zones of South Africa and South America. The relative humidity values are computed with respect to water vapor saturation pressure. (At an altitude of $400 \mathrm{hPa}$, the ratio of saturation pressure over ice to that over water is about 0.8.)

\section{WV wind fields}

\section{a. Meteosat-5}

The method for deriving Meteosat-5 WV winds at ESOC is described by Laurent (1993) and Holmlund (1993). The height assignment of the WV winds follows two approaches. (a) If the target area for tracking 


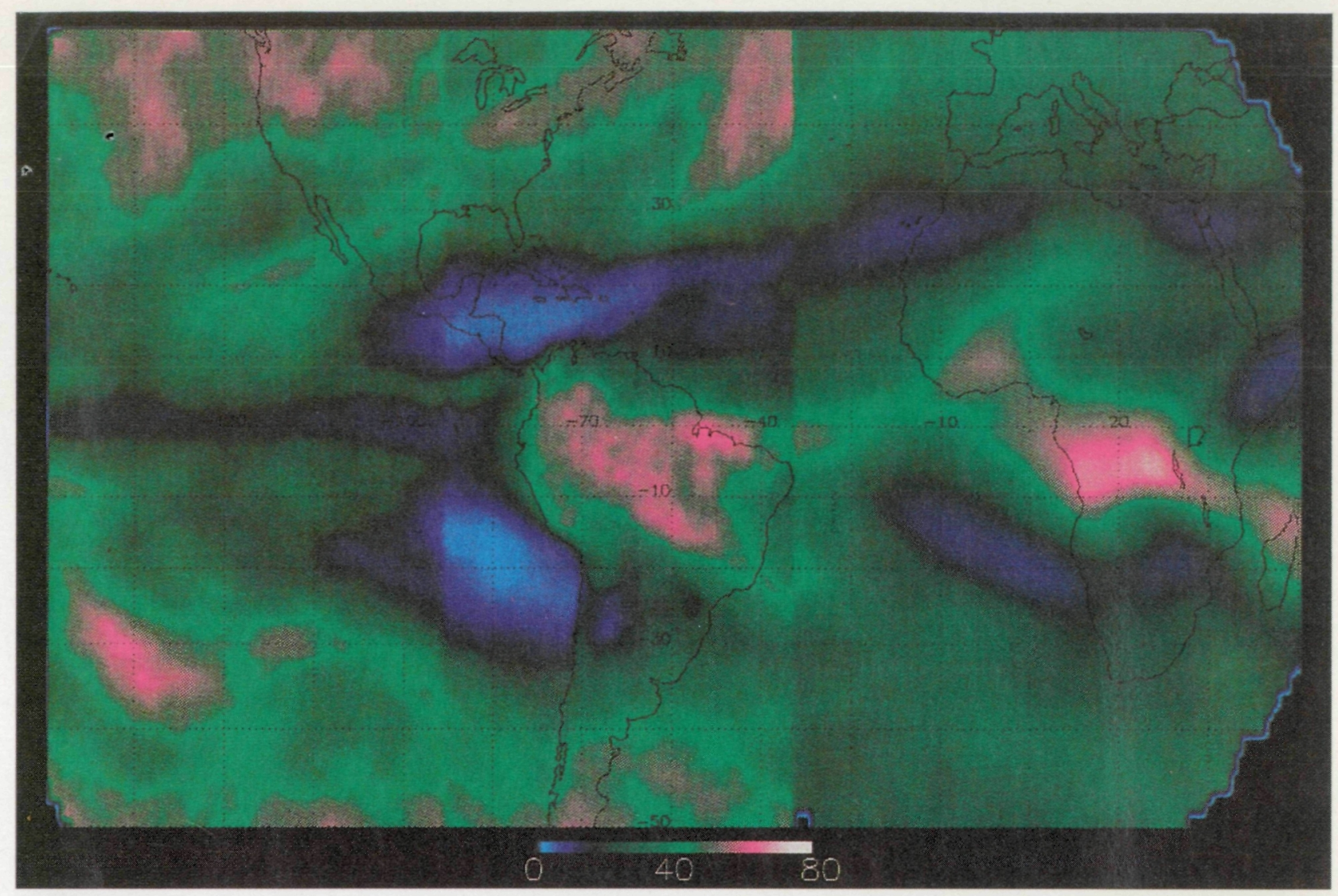

FIG. 3. The monthly mean UTH for March 1994 from all three satellites. Values have been averaged to roughly a $1^{\circ}$ lat-long grid. The adjusted GOES-7 UTH merges very well with the Meteosat-3 UTH without noticeable discontinuity. On the other hand, the merge line between Meteosat-3 and Meteosat-5 is distinguishable, primarily because different UTH retrieval procedures are used.

the displacement contains cloudy features, the height assignment is the same as for the high-level infrared cloud motion winds (Schmetz et al. 1993). Opaque clouds are assigned to the pressure level where the brightness temperature fits a short-term forecast profile; semitransparent clouds are assigned to a pressure level using the IR and WV bands. (b) If the target area is cloud free, the coldest $25 \%$ of the WV pixels in a target area is used to compute a brightness temperature for the height assignment (Laurent 1993). The brightness temperatures are converted to pressure heights via short-term forecast temperature profiles. The adequacy of this approach has been verified in detailed comparisons with rawinsondes (Holmlund 1993).

\section{b. Meteosat-3 and GOES-7}

Water vapor wind sets derived at CIMSS from Meteosat-3 and GOES-7 are processed from hourly full-disk images using fully automated procedures [see Merrill et al. (1991) for further details]. Potential targets are identified by maximum brightness temperature gradients exceeding predetermined thresh- olds within a selected target region. Heights are assigned using the WV band brightness temperature of the coldest $25 \%$ of the target region matched to a short-term forecast profile. The vertical distribution of the pressure heights is typically in the range of 200-500 $\mathrm{hPa}$ with a peak near $300 \mathrm{hPa}$. As a final step, an objective quality control procedure is invoked once the wind vector field has been derived (Hayden and Velden 1991). Statistical comparisons between WV wind vectors derived at CIMSS and collocated Western Hemisphere rawinsondes show rms differences that are close to those of current operational upper-air cloud-drift winds (Velden et al. 1994). Water vapor winds have also been shown to have positive impact on NWP (Velden 1995).

\section{c. Mean WV wind field for March 1994}

The daily WV winds produced by both CIMSS $\left(2 \mathrm{~d}^{-1}\right)$ and ESOC $\left(4 \mathrm{~d}^{-1}\right)$ for March 1994 are used to calculate a monthly mean field by averaging all wind vectors contained within $2.0^{\circ}$ lat-long geographical regions (grid points). A monthly mean gridpoint motion vector value is considered to be valid when the num- 


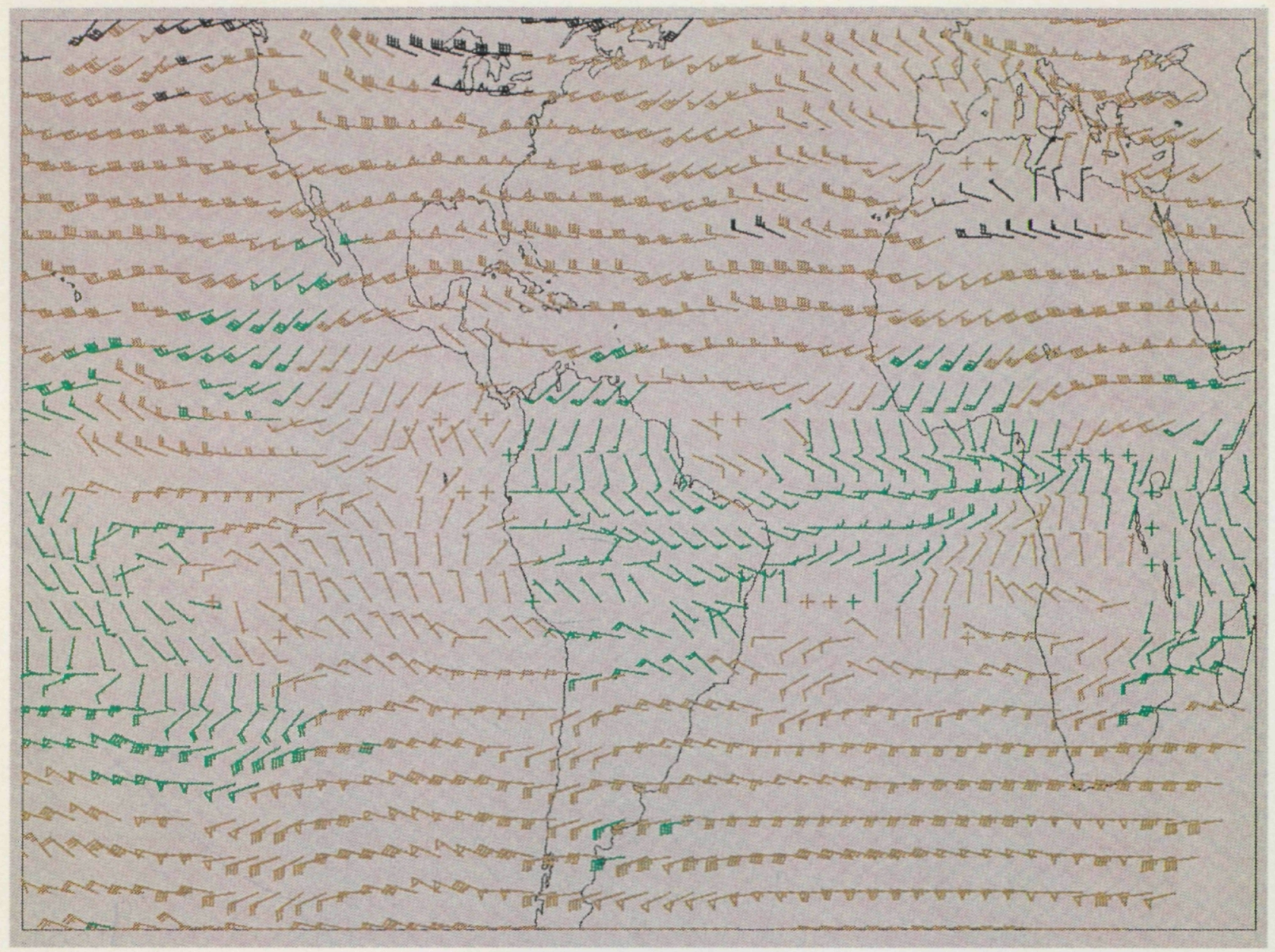

FIG. 4. The mean WV wind vectors displayed in kts for March 1994. (Vectors every $4^{\circ}$ are shown.) Blue indicates vectors with pressures greater than $400 \mathrm{hPa}$; brown indicates vectors with pressures between 300 and $399 \mathrm{hPa}$; and green corresponds to vectors with pressures less than $300 \mathrm{hPa}$.

ber of observations exceeds 20. (Only grid points near the limb fail this criterion.) The monthly mean gridpoint height value is calculated by averaging individual vector height assignments. Figure 4 illustrates that the mean WV wind vectors show good consistency and the heights are confined to adequately describe the upper-tropospheric layer mean flow.

\section{UTH and WV wind field divergence}

To associate the observed upper-tropospheric circulation with the observed UTH, divergence fields are computed with a finite-difference scheme from the monthly mean WV winds. The dashed contours in Fig. 5 indicate mean divergent areas that are often associated with water vapor emerging from source regions (deep convective areas). For example, the pattern in the UTH over South America is clearly correlated with the divergence field.
The solid contours in Fig. 5 correspond to uppertropospheric convergence, most prominent over subtropical subsidence regions. The observed convergence values appear quite reasonable: assuming that the convergence takes place in a layer of about $150 \mathrm{hPa}$, the associated subsidence is about $60 \mathrm{hPa} \mathrm{d}^{-1}$, which compares favorably to the observed subsidence in the trade wind regions (Betts and Ridgway 1989).

A comment is warranted concerning the height variation of the WV winds. Inspection of Fig. 4 shows that the monthly mean altitudes of the $\mathrm{WV}$ winds range approximately between 250 and $350 \mathrm{hPa}$. The spatial changes are smooth, and the standard deviation at a geographical location is only $30-70 \mathrm{hPa}$. Thus, the computed divergence/convergence patterns do not correspond to a constant pressure surface but to a smoothly varying surface with higher altitudes in the convective area than in the subsidence regions.

One should not expect a perfect match between UTH and wind field divergence. Over tropical areas 


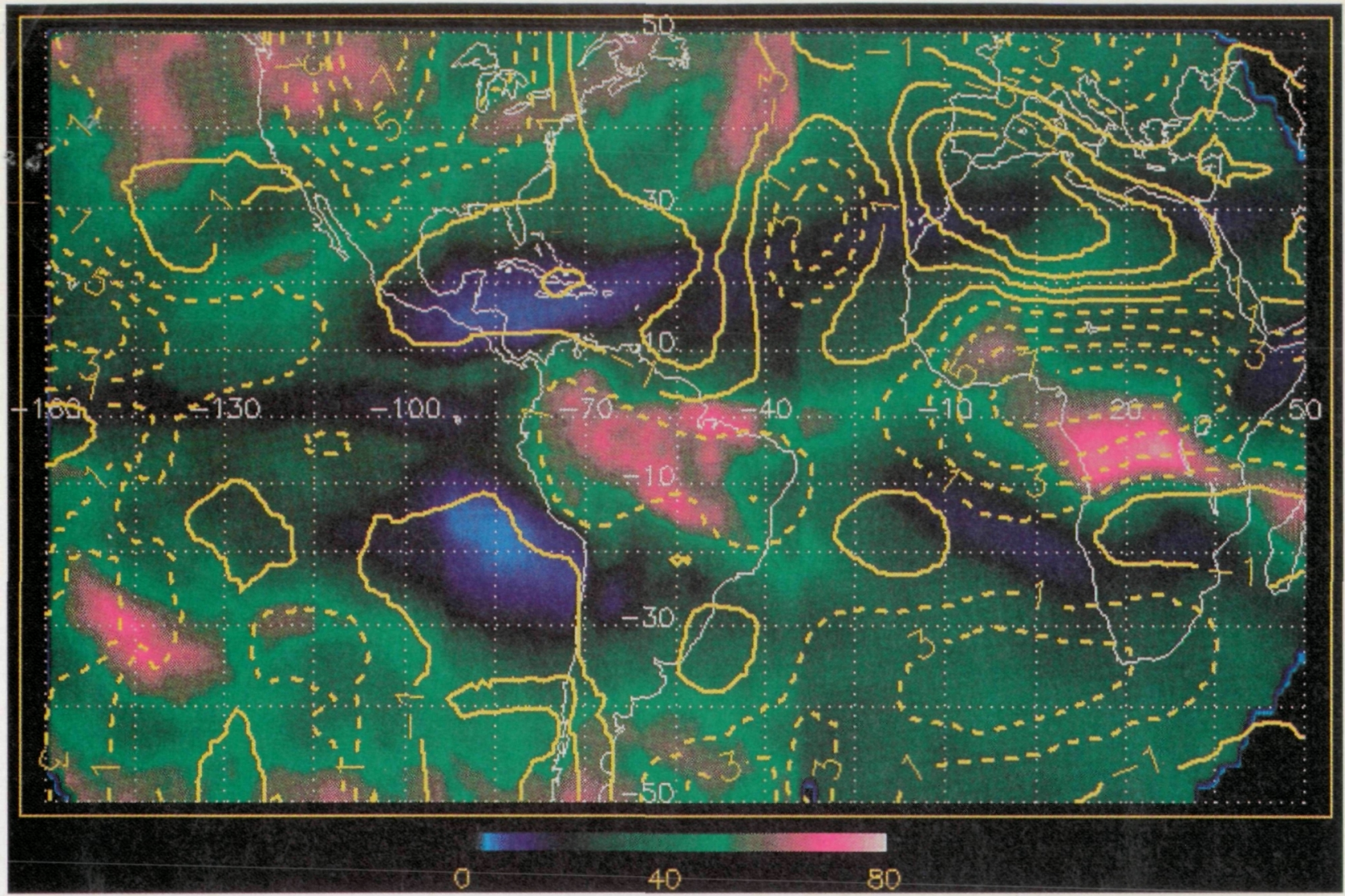

FIG. 5. Simultaneous display of the upper-level convergence fields (derived from WV winds) and the observed UTH for March 1994. The dashed contours correspond to divergent areas in the upper troposphere; solid contours represent convergence. Contours are shown in intervals of $2 \times 10^{-6} \mathrm{~s}^{-1}$. The UTH is displayed as a color image with a relative humidity scale displayed. (Blue is dry, green is in the middle, and red is moist.)

especially, the UTH maxima are associated with convection usually forced by surface heating and/or convergence. On the other hand, the WV wind field is primarily representative of upper-level large-scale flow, with secondary influence from large convective regimes. The UTH and divergence maxima should be in close proximity, but they need not be aligned. An example is evident in Fig. 5 over Africa, where the UTH maximum is shifted to the south of the wind divergence maximum. The position of the divergence maximum appears to be influenced by the entrance region of the subtropical jet over Africa. However, over the South American continent, where no jet stream is observed, the divergence maximum matches well with the UTH maximum.

The congruence of the UTH and WV wind divergence patterns suggests a physical relationship in which the UTH patterns are largely influenced by upper-tropospheric transport. Picon and Desbois (1990) indicate that the upper-tropospheric humidity field is chiefly determined by large-scale dynamics. The clear-sky UTH climatology of van de Berg et al. (1991) corroborates this statement, as the geographi- cal distribution of monthly UTH fields is reminiscent of the Hadley and Walker circulation patterns. Soden and Bretherton (1993) also point out that the lower troposphere is the prime source of upper-tropospheric water vapor and that deep convection is the transport mechanism. However, the novel message of this study is that both UTH and the upper-tropospheric dynamics can be inferred simultaneously from geostationary satellites. As such, the satellite-derived UTH and WV winds constitute a valuable source for climatological diagnostics and for comparison with large-scale circulation models.

\section{Summary}

Monthly mean fields of the upper-tropospheric relative humidity (UTH) and water vapor (WV) winds from Meteosat-3, Meteosat-5, and GOES-7 WV band observations have been analyzed for March 1994. The fields provide a concise description of monthly mean upper-tropospheric moisture and motion fields. The scope of the present study is a qualitative demonstra- 
tion that UTH and upper-tropospheric dynamics are directly observable on a nearly global scale; a future quantitative analysis will include a much larger dataset.

The comparison of the March 1994 UTH and WV wind fields shows the influence of atmospheric dynamics on the distribution of the upper-tropospheric moisture. Since upper-tropospheric moisture is an important factor in the atmospheric greenhouse effect, the satellite-observed fields of UTH and WV wind are important for climate model validation. Most of the previous comparisons of climate models with observed moisture fields used only total precipitable water vapor observations over the oceans. Since lowlevel moisture dominates precipitable water, such comparisons do not validate the upper-tropospheric moisture fields in models.

This study using satellite measurements docu-ments an example where the upper-tropospheric moisture distribution is strongly influenced by the large-scale dynamics. Longer-term datasets of both UTH and $W V$ winds covering seasonal and interannual variations would be useful for comparison with climate models and for validation of the dynamical aspects of the models. Such a study could help reveal how the large-scale circulation would be affected by climate change.

This study also suggests that use of WV winds should improve representation of upper-tropospheric moisture fields in numerical models. This may be important to medium-range forecasts since the radiative heating/cooling critically depends on upper-tropospheric moisture. The importance is amplified by the fact that the moisture field influences the cloud field, which has an even larger effect on radiative heating/ cooling and also implies energy transformations through phase changes.

Both UTH and WV winds could be very desirable contributors to a climate-monitoring system. The monitoring of both quantities will soon be possible on a nearly global basis since future geostationary satellites from China and Japan will also be capable of radiance measurements in the WV band. Further detailed studies are needed to fully understand the features in simultaneous UTH and WV wind fields. A continuous operational derivation of the two products would provide the basis for investigation of seasonal and interannual variations.

\section{References}

Betts, A., and W. Ridgway, 1989: Climatic equilibrium of the convective boundary layer over the tropical ocean. J. Atmos. Sci, 46, 2621-2641.
Del Genio, A. D., W. Kovari Jr., and M.-S. Yao, 1994: Climatic implications of the seasonal variation of upper tropospheric water vapor. Geophys. Res. Lett., 21, 2701-2704.

Fischer, H., N. Eigenwillig, and H. Mueller, 1981: Information content of Meteosat and Nimbus/THIR water vapor channel data: Altitude association of observed phenomena. J. Appl. Meteór., 20, 1344-1352.

Hayden, C., 1988: GOES-VAS simultaneous temperature-moisture retrieval algorithm. J. Appl. Meteor., 27, 705-733.

$\longrightarrow$, and C. Velden, 1991: Quality control and assimilation experiments with satellite-derived wind estimates. Preprints, Ninth Conf. on Numerical Weather Prediction, Denver, CO, Amer. Meteor. Soc., 19-23.

Holmlund, K., 1993: Operational water vapor wind vectors from Meteosat imagery data. Proc. Second Int. Winds Workshop, Tokyo, Japan, European Organization for the Exploitation of Meteorological Satellites, 77-85.

Inamdar, A. K., and V. Ramanathan, 1994: Physics of greenhouse effect and convection in warm oceans. J. Climate, 7, 715-731.

Laurent, $H_{\text {., }}$ 1993: Wind extraction from Meteosat water vapor channel image data. J. Appl. Meteor., 32, 1124-1133.

Menzel, W. P., S. Wanzong, S. Nieman, F. Wu, C. Velden, and J. Schmetz, 1994: Comparison of GOES and Meteosat Calibration and Products. Proc. 10th Meteosat Scientific Users Conf., Cascais, Portugal, European Organization for the Exploitation of Meteorological Satellites, 71-82.

Merrill, R., P. Menzel, W. Baker, J. Lynch, and E. Legg, 1991: A report on the recent demonstration of NOAA's upgraded capability to derive cloud motion satellite winds. Bull. Amer. Meteor. Soc., 66, 1111-1115.

Picon, L., and M. Desbois, 1990: Relation between METEOSAT watervapor radiance fields and large scale tropical circulation. $J$. Climate, 3, 865-874.

Schmetz, J., and O. M. Turpeinen, 1988: Estimation of the upper tropospheric relative humidity field from METEOSAT water vapor image data. J. Appl. Meteor., 27, 889-899.

_- and L. van de Berg, 1994: Upper tropospheric humidity observations from Meteosat compared with short-term forecast fields. Geophys. Res. Lett., 21, 573-576.

- K. Holmlund, J. Hoffman, B. Strauss, B. Mason, V. Gaertner, A. Koch, and L. van de Berg, 1993: Operational cloud-motion winds from Meteosat infrared images. J. Appl. Meteor., 32, 1206-1225.

Soden, B., and F. P. Bretherton, 1993: Upper tropospheric relative humidity from GOES 6.7 micron channel: Method and climatology for July 1987. J. Geophys. Res., 98, 16 669-16 688.

Stephens, G. L., D. A. Randall, I. L. Wittmeyer, and D. A. Dazlich, 1993: The Earth's radiation budget and its relation to atmospherichydrology 3. Comparison of observations over the oceans with a GCM. J. Geophys. Res., 98(D3), 4931-4950.

van de Berg, L., A. Pyomjamsri, and J. Schmetz, 1991: Monthly mean upper-tropospheric humidities in cloud-free areas from METEOSAT observations. Int. J. Climatol., 11, 819-826.

Velden, C., 1995: Winds derived from geostationary satellite moisture channel observations: Applications and impact on numerical weather prediction. Meteor. Atmos. Phys., in press.

- S. Nieman, and S. Wanzong, 1994: Investigation of water vapor motion winds from geostationary satellites. Preprints, Seventh Conf. on Satellite Meteorology and Oceanography, Monterey, CA, Amer. Meteor. Soc., 360-363.

Weinreb, M. P., R. Xie, J. H. Lienesch, and D. S. Crosby, 1989: Destriping GOES images by matching empirical diștribution functions. Remote Sens. Environ., 29, 185-195.

Wu, X., J. J. Bates, and S. J. S. Khalsa, 1993: A climatology of the water vapor band brightness temperatures from NOAA operational satellites. J. Climate, 6, 1282-1300. 